\title{
Molecular Epidemiology of Multidrug-Resistant Tuberculosis, New York City, 1995-1997
}

\author{
Sonal S. Munsiff, ${ }^{\dagger} \dagger$ Trina Bassoff, ${ }^{*}$ Beth Nivin, ${ }^{*}$ Jiehui Li, ${ }^{*}$ Anu Sharma, ${ }^{*}$ Pablo Bifani,‡ \\ Barun Mathema,‡ Jeffrey Driscoll,§ and Barry N. Kreiswirth
}

\begin{abstract}
From January 1, 1995, to December 31, 1997, we reviewed records of all New York City patients who had multidrug-resistant tuberculosis (MDRTB); we performed insertion sequence (IS) 6110-based DNA genotyping on the isolates. Secondary genotyping was performed for low IS6110 copy band strains. Patients with identical DNA pattern strains were considered clustered. From 1995 through 1997, MDRTB was diagnosed in 241 patients; 217 (90\%) had no prior treatment history, and 166 (68.9\%) were born in the United States or Puerto Rico. Compared with non-MDRTB patients, MDRTB patients were more likely to be born in the United States, have HIV infection, and work in health care. Genotyping results were available for 234 patients; $153(65.4 \%)$ were clustered, 126 (82.3\%) of them in eight clusters of $\geq 4$ patients. Epidemiologic links were identified for $30(12.8 \%)$ patients; most had been exposed to patients diagnosed before the study period. These strains were likely transmitted in the early 1990s when MDRTB outbreaks and tuberculosis transmission were widespread in New York.
\end{abstract}

W idespread transmission of multidrug-resistant Mycobacterium tuberculosis (MDRTB) strains occurred during the epidemic of the 1980s and early 1990s in New York City. Outbreaks were identified in many New York City hospitals and subsequently in New York State correctional facilities. Many of these outbreaks were associated with one strain (known as the "W" strain of TB) that was resistant to isoniazid, rifampin, ethambutol, and streptomycin and usually to kanamycin (1-5). However, other multidrug-resistant (MDR) strains were associated with outbreaks and nosocomial transmission during these years (6-8). Previous molecular epidemiology surveys in New York City showed that MDRTB was associated with clustered M. tuberculosis strains, which suggests recent transmission of the organism (9-11). The incidence of tuberculosis (TB) and MDRTB has been decreasing rapidly in New York City since 1992, when an enhanced Tuberculosis Control Program was implemented. The number of TB cases decreased 21.5\% by 1994 (from 3,811 in 1992 to 2,995 in 1994), and MDRTB cases decreased 60\% (from 441 to 176$)(12,13)$. Since 1994, no outbreaks of MDRTB have been documented in the city.

To better understand the epidemiology of MDRTB, the New York City Tuberculosis Control Program began DNA genotyping of MDRTB strains from new cases in 1995. The objectives were to provide descriptive molecular epidemiology of MDRTB cases in the city during 1995-1997 and to

*New York City Department of Health, New York, New York, USA; †Centers for Disease Control and Prevention, Atlanta, Georgia, USA; $\ddagger$ Public Health Research Institute TB Center, Newark, New Jersey, USA; and §Wadsworth Center, Albany, New York, USA identify predominant MDR strains present during these years, as well as the extent and risk factors for clustering among these cases.

\section{Methods}

\section{Patient Selection}

All patients with MDRTB (M. tuberculosis isolate resistant to at least isoniazid and rifampin) confirmed as TB cases from January 1, 1995, to December 31, 1997, in New York City were included. Demographic and clinical data were obtained from the New York City Tuberculosis Case Registry. The Registry's data were obtained from patient interviews and medical record reviews at the treatment or residential facilities by trained case managers using standardized data collection instruments and from contact investigations for each pulmonary case.

Susceptibility results were reviewed for the following TB treatment drugs: isoniazid, rifampin, pyrazinamide, ethambutol, streptomycin, and rifabutin (first-line drugs) and fluoroquinolone (usually ciprofloxacin or oflaxacin), kanamycin or amikacin, capreomycin, ethionamide, para-aminosalicylic acid, and cycloserine (second-line drugs). Susceptibility tests were done by Bactec radiometric method (Becton Dickinson and Co., Sparks, NY) for first-line drugs, except rifabutin (14), for most isolates and with standard proportion method with Middlebrook 7H10 media for both first- and second-line drugs for all isolates (15). Most of these tests were conducted at two reference laboratories, the New York City Department of Health and the New York State Department of Health, Wadsworth Center. 
As part of routine surveillance, we reviewed the clinical histories of all pulmonary TB patients who had a negative acidfast bacilli smear and only one positive $M$. tuberculosis isolate from a respiratory source. This review was to determine the accuracy of the culture result and to rule out laboratory error. If laboratory error was suspected for other types of specimens, clinical and laboratory data for patients were reviewed. Laboratory error was defined as a false-positive M. tuberculosis culture result that was caused by specimen mislabeling or laboratory cross-contamination, as evaluated by a described method (10). These patients were not counted as having verified cases of TB and were excluded from the analysis.

\section{Definitions}

Patients were defined as having had prior treatment for TB if 1) drug-susceptible M. tuberculosis isolates were identified before the drug-resistant isolates that qualified the patients for this study; 2) they had documentation of previous TB disease or treatment; or 3) they had received $>30$ days of treatment with anti-TB drugs before collection of the specimen that grew MDR M. tuberculosis.

Patients were considered HIV seropositive when a positive HIV antibody test result was documented in the medical record or when AIDS was diagnosed before the TB diagnosis. The MDRTB diagnosis date was defined as the collection date of the first specimen from which an MDR M. tuberculosis isolate was cultured. Homelessness was defined as being in a public or private shelter or having no address at the time of the MDRTB diagnosis. Information about injection drug use within the 12 months before diagnosis was elicited from direct patient interviews and medical record reviews.

\section{Epidemiologic Investigations}

Trained case managers obtained information about suspected and confirmed nosocomial and community exposure from patient interviews, contact investigations, and medical record reviews at the treatment or residential facilities. Probable nosocomial transmission was considered if the newly infected patient was in the same section of an institution as another patient who had an identical M. tuberculosis strain and was infectious (i.e., the patient had a positive culture from a respiratory site) at least 30 days before disease onset in the newly infected patient.

Community transmission was considered probable if either of the following occurred: 1) A patient was exposed to another patient who had the identical M. tuberculosis strain and was infectious (i.e., had a positive culture from a respiratory site) at least 30 days before disease onset in the subsequent patient. The exposure would have occurred in a home, single-room occupancy hotel, homeless shelter, or another noninstitutional setting. 2) The patient named another patient as a contact whose $M$. tuberculosis isolate had the same DNA pattern or who had MDRTB, but DNA genotyping result was not available.

Transmission could have been from a patient whose condition was diagnosed before the study period. If evidence of nosocomial or community transmission was found, patients had an epidemiologic link. The source patient was not considered to have an epidemiologic link.

During 1995 through 1996, nosocomial transmission was suspected at a hospital where the same MDR strain (i.e., identical insertion sequence [IS] 6110 band patterns) was found in six patients. Hospital floor, ward, and bed information and computerized outpatient clinic records from 1990 to 1996 were analyzed for temporal and spatial overlap among these patients. Medical records were reviewed for patient breaches of isolation protocol during hospitalization. Additional social and demographic information was collected through questionnaires. Specifically, patients were asked with whom and where they spent considerable time, and names of additional social contacts were requested. Patients were asked where and how they thought they had been exposed to TB.

\section{IS6110 DNA Genotyping and Other Molecular Studies}

From 1995 through 1997, one M. tuberculosis isolate from each patient with MDRTB in New York City was sent to the Public Health Research Institute Tuberculosis Center, where DNA fingerprint analysis, based on IS6110 Southern blot hybridization pattern, was performed by using a standardized protocol (16). The Southern hybridization patterns were compared on a Sun Sparc5 Workstation (Sun MicroSystems, Santa Clara, CA), using Bio Image Whole Band Analyzer software version 3.4 (Bio Image, Ann Arbor, MI). Previously described methods were used to classify isolates (17). IS6110 banding patterns, which were similar to a parent strain but differed by one or two hybridization bands, were denoted by the addition of a number to the cluster letter (e.g., W, W1, P, or P1).

Secondary genotyping was performed by using spacer oligonucleotide typing (spoligotyping) and DNA sequencing of target gene regions that confer drug resistance. Spoligotyping and DNA sequencing of target gene regions used previously described methods (18-21).

If M. tuberculosis isolates had identical IS6110 band patterns, they were considered clustered. However, identical IS6110 patterns with less than six bands were not considered clustered, unless secondary DNA analysis confirmed a match, as noted in the results.

\section{Data Analysis}

To examine how MDR patients differed from non-MDR patients, study subjects were compared to persons who had culture-positive TB diagnosed during the same period but were not included in this study. Descriptive analysis was performed for all study patients according to drug resistance patterns, DNA patterns, prior TB treatment, social and demographic variables, and evidence of nosocomial and community transmission. The Wilcoxon rank-sum test was used to compare medians of continuous variables, and the Pearson chisquare test was used to compare categorical data. Unconditional logistic regression was used to assess crude odds ratios and $95 \%$ confidence intervals for the association between 
potential risk factors and clustering. Statistical Analysis System Software (Release 8.01, SAS Institute, Inc., Cary, NC) was used for all data analyses. Statistical significance was set at a two-sided $5 \%$ level.

\section{Results}

From 1995 through 1997, a total of 6,228 cases of TB were confirmed in New York City. Cultures from 5,136 (82.4\%) persons were positive for $M$. tuberculosis. Of these, susceptibility results were available for 4,955 (96.5\%); 241 (4.9\%) persons had MDRTB. Findings of MDR for 11 additional isolates resulted from laboratory error (10 sputum and 1 urogenital); they were excluded from further analyses. The 241 patients made up 4.4\% (106 of 2,445), 3.9\% (81 of 2,053), and $3.1 \%$ (54 of 1,730) of all verified patients who had TB from 1995, 1996, and 1997, respectively. Table 1 presents a comparison of the demographic characteristics of these patients to those of culture-positive non-MDRTB patients from the same time period in New York City for whom drug susceptibility results were available. Compared with patients with culturepositive non-MDRTB during the same period, MDR patients were more likely to be born in the United States, have HIV infection, and be health-care workers, homeless, and injection drug users. MDR patients were more likely to have respiratory specimens positive for acid-fast bacilli and were less likely to be Asian. By further stratification, none of Asian MDRTB patients were born in the United States, and $68.7 \%$ of U.S.born MDRTB patients were HIV infected.

Strains were resistant to a median number of 6 drugs (range 2-10). Eight (3.3\%) patients had strains of M. tuberculosis that were resistant to isoniazid and rifampin only, and 146 $(60.6 \%)$ had isolates that were also resistant to one or more second-line anti-TB drugs. Most of these strains were also resistant to rifabutin. Twenty-four (10\%) patients had received prior treatment for TB. Compared with patients who had no prior treatment, patients who had received such treatment were significantly older (median age 46 years vs. 41 years, $p=0.010$ ) and had less drug resistance (median 5 drugs versus 6, $\mathrm{p}=0.042$ ). Patients with prior treatment were less likely to be born in the United States $(45.8 \%$ vs. $71.4 \%, \mathrm{p}=0.001)$ and were less likely to be HIV infected (33.3\% vs. 55.3\%, $\mathrm{p}=0.041$ ). Patients who had received prior treatment did not differ from those who had no prior treatment according to gender, race or ethnicity, occupation, and histories of alcohol or drug abuse and homelessness.

\section{DNA Genotyping Analysis}

Of 241 MDR patients, 234 (97\%) had IS6110 fingerprint patterns. Ninety-two different patterns were identified (band range 2-22). Thirty-six (15.4\%) of 234 isolates had patterns with five or fewer IS6110 bands. Five were in one cluster, the $\mathrm{C}$ strain, and all had the same spoligotype (700036777760731). Two were clustered as a four-band strain with the same spoligotypes, and three other strains had unique genotypes. Twenty-six strains had an identical two-band
Table 1. Comparison of social and demographic characteristics of multidrug-resistant (MDR) tuberculosis patients and non-multidrug-resistant tuberculosis patients, New York City, 1995-1997

\begin{tabular}{|c|c|c|c|c|c|}
\hline \multirow[b]{2}{*}{ Characteristic } & \multicolumn{2}{|c|}{$\operatorname{MDR}(n=241)$} & \multicolumn{2}{|c|}{$\begin{array}{c}\text { Non-MDR } \\
(n=4,714)\end{array}$} & \multirow[b]{2}{*}{$\mathrm{p}$ value } \\
\hline & No. & $\%$ & No. & $\%$ & \\
\hline \multicolumn{6}{|l|}{ Yr of diagnosis } \\
\hline 1995 & 106 & 44.0 & 1,816 & 38.5 & 0.0898 \\
\hline 1996 & 81 & 33.6 & 1,586 & 33.6 & 0.9912 \\
\hline 1997 & 54 & 22.4 & 1,312 & 27.8 & 0.0660 \\
\hline Median age (range), yrs & \multicolumn{2}{|c|}{$41(5-85)$} & \multicolumn{2}{|c|}{$41(0-100)$} & 0.3071 \\
\hline Male sex & 147 & 61.0 & 3,020 & 64.1 & 0.3333 \\
\hline U.S.-born & 166 & 68.9 & 2,483 & 52.7 & $<0.001$ \\
\hline \multicolumn{6}{|l|}{ HIV serostatus } \\
\hline Positive & 128 & 53.1 & 1,478 & 31.4 & $<0.001$ \\
\hline Negative & 75 & 31.2 & 1,759 & 37.3 & 0.0521 \\
\hline Unknown & 38 & 15.8 & 1,477 & 31.3 & $<0.001$ \\
\hline \multicolumn{6}{|l|}{ Race/ethnicity } \\
\hline Asian & 24 & 10.0 & 817 & 17.3 & 0.0029 \\
\hline Hispanic & 75 & 31.1 & 1,266 & 26.9 & 0.1461 \\
\hline Black non-Hispanic & 105 & 43.6 & 2,089 & 44.3 & 0.8200 \\
\hline White non-Hispanic & 37 & 15.4 & 542 & 11.5 & 0.0692 \\
\hline Health-care worker & 15 & 6.2 & 109 & 2.3 & 0.0001 \\
\hline Homeless & 28 & 11.6 & 344 & 7.3 & 0.0130 \\
\hline Injection drug user & 33 & 13.7 & 275 & 5.8 & $<0.001$ \\
\hline \multicolumn{6}{|l|}{ Disease site } \\
\hline Pulmonary only & 176 & 73.0 & 3,397 & 72.1 & 0.7440 \\
\hline Extra-pulmonary only & 35 & 14.5 & 894 & 19.0 & 0.0848 \\
\hline Pulmonary + extra-pulmonary & 30 & 12.5 & 423 & 9.0 & 0.0679 \\
\hline Specimen AFB smear-positive $^{a}$ & 141 & 68.5 & 2,129 & 55.7 & 0.0003 \\
\hline Cavitary lesion(s) ${ }^{\mathrm{b}}$ & 47 & 22.8 & 740 & 19.4 & 0.2247 \\
\hline
\end{tabular}

${ }^{a}$ Respiratory specimen during the 30 days after initial specimen for bacteriologic test was taken. Excludes those who had only extrapulmonary disease.

${ }^{b}$ Excludes those with extrapulmonary disease only.

IS6110 pattern designated as $\mathrm{H} ; 25$ of the 26 were resistant to pyrazinamide. All 17 with available results had identical spoligotypes (777776777760601); 18 of the 20 strains that were tested had identical pncA genotype (Nt70; G deletion). One pyrazinamide-susceptible strain had the wild-type $p n c A$ genotype, and one resistant strain had a different $p n c A$ genotype (139; GTG $>$ GCG, Val $>$ Ala). On the basis of the results, 18 of the $26 \mathrm{H}$ strains were considered clustered. Thus, 25 of the 36 isolates with low IS6110 copies were considered clustered.

Of 234 patients with DNA results, 153 (65.4\%) were grouped into 19 clusters: 6 clusters with 2 cases each; 5 clusters with 3 cases each; and 8 different clusters with 4, 5, 6, 7, 13, 14, 18, and 59 cases each. The eight clusters had 126 (52.2\%) of 241 MDRTB patients from the study period. Table 2 shows the distribution of these eight strains during 1995 
TUBERCULOSIS GENOTYPING NETWORK

Table 2. Social and demographic characteristics of patients in predominantly multidrug-resistant tuberculosis clusters, New York City, 1995-1997

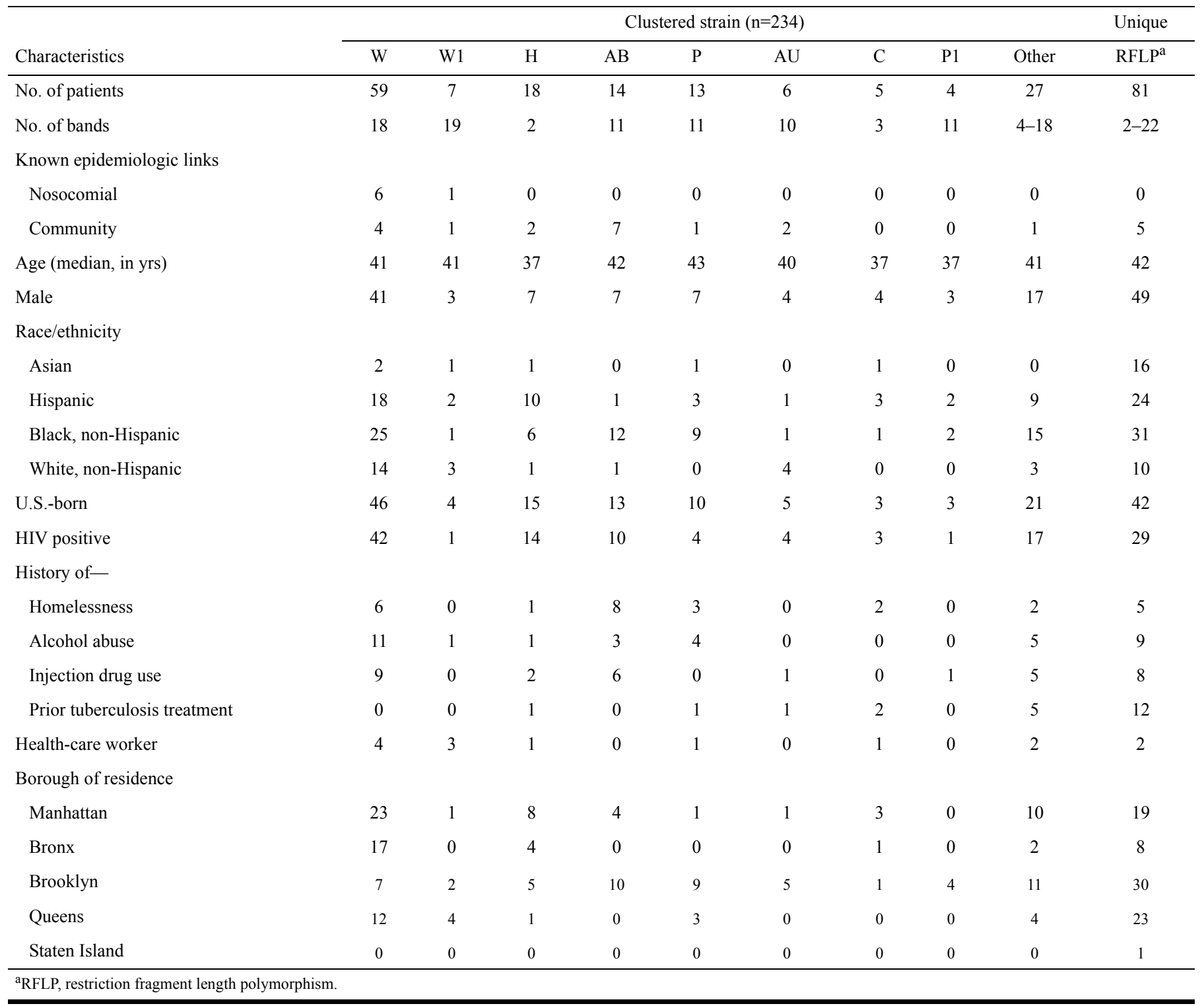

through 1997 with social, demographic, and epidemiologic link information. Figure 1 shows the geographic distribution, and Figure 2 shows the IS6110 patterns of these eight strains.

Epidemiologic links were identified for 30 (12.8\%) of the 234 patients with genotyping results; most had been exposed to patients diagnosed before the study period. Twenty-five (19.8\%) of 153 patients clustered by DNA genotyping were epidemiologically linked; $18(72 \%)$ had probable community transmission, and 7 (28\%) had probable nosocomial transmission. All nosocomial links were to patients whose conditions were diagnosed before the study period. Seven community transmission links were to patients from the study period, and 11 were to patients whose diseases were diagnosed before the study period. Epidemiologic links of community transmission were identified for $5(6.2 \%)$ of 81 nonclustered patients; all were links to persons whose conditions were diagnosed before the study period. Of the 23 community links, 3 were to house- hold members, 4 to nonhousehold relatives, and 7 to friends. One was linked to another case in a single-room occupancy hotel; seven were linked in a crack den, and one had an unknown exposure site.

Table 3 shows a comparison of patients clustered by DNA analysis to those nonclustered according to various demographic and clinical characteristics. Factors significantly associated with clustering were HIV infection and birth in the United States. There was no difference in proportion clustered by year. Patients with histories of prior treatment and Asian patients were significantly less likely (odds ratio $[\mathrm{OR}]=0.40$, $95 \%$ confidence interval $[\mathrm{CI}]=0.17$ to 0.98 and $\mathrm{OR}=0.18$, $95 \%$ CI-0.06 to 0.53 , respectively) to be in a cluster. Patients in clusters were 3 times more likely to have epidemiologic links than those not in clusters. In a subanalysis that included only non-U.S.-born patients who had a known date of entry to the United States, clustering was significantly associated 


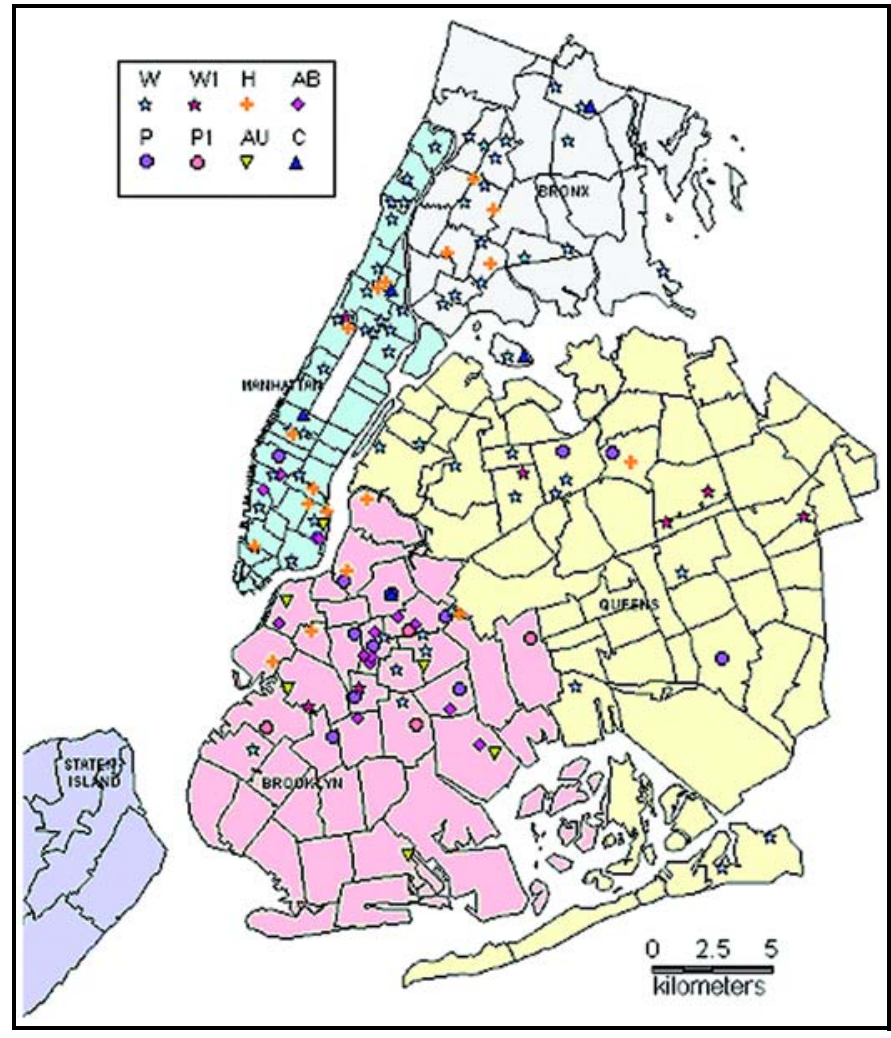

Figure 1. Geographic distribution of patients in major multidrug-resistant tuberculosis clusters, New York City, 1995-1997.

$(\mathrm{OR}=1.09,95 \% \mathrm{CI}=1.02,1.16 ; \mathrm{p}=0.01)$ with longer time of residence in the United States.

\section{Epidemiology of Predominant MDR Strains}

Fourteen patients in this study had an 11-band strain (AB). Six of these patients were diagnosed at a single medical facility in Brooklyn, New York. At the time of diagnosis, five of these persons reported a home address in the same health district as the medical facility. Although two patients were hospitalized at the medical facility when transmission could have occurred, hospital inpatient and outpatient records showed that nosocomial transmission was unlikely because of the room locations and documented adherence to isolation protocol.

Our study showed the following characteristics for patients in the AB cluster: $92.9 \%$ were born in the United States, $71.4 \%$ were infected with HIV, $85.7 \%$ were non-Hispanic black, $42.8 \%$ used injection drugs, and $100 \%$ had no prior treatment for TB. These patients reported home addresses from only two of five boroughs in New York City, 10 (71.4\%) in Brooklyn and 4 (28.6\%) in Manhattan. However, 57\% were homeless. Five patients agreed to additional interviews; six patients had died, and three patients could not be located. On the basis of the additional interviews and available data from initial interviews, 7 of these 14 patients had community transmission links. Two of these links were found through standard contact investigations, and five were disclosed by the additional patient interviews. Three patients had close contacts with two patients who had the AB strain in 1992; four fre- quented the same crack den in the neighborhood of the medical facility before their TB diagnosis. The remaining seven patients had no history of contact with persons who had the AB strain.

The largest cluster was from the W strain-59 patients representing almost $25 \%$ of the 241 MDRTB patients in the 3 years. This strain caused a well-documented multi-institutional outbreak in New York City from 1990 through 1993 (1-5). Strain W1, which was isolated in seven patients, is a variant of the $\mathrm{W}$ strain. It has an additional IS6110 copy and is part of the W strain outbreak $(4,5)$. Forty percent (12 of 30) of the epidemiologic links in this cohort were to patients with these two strains. Seven (46.7\%) of the 15 health-care workers had either the W strain (4 cases) or the W1 strain (3 cases). However, epi-

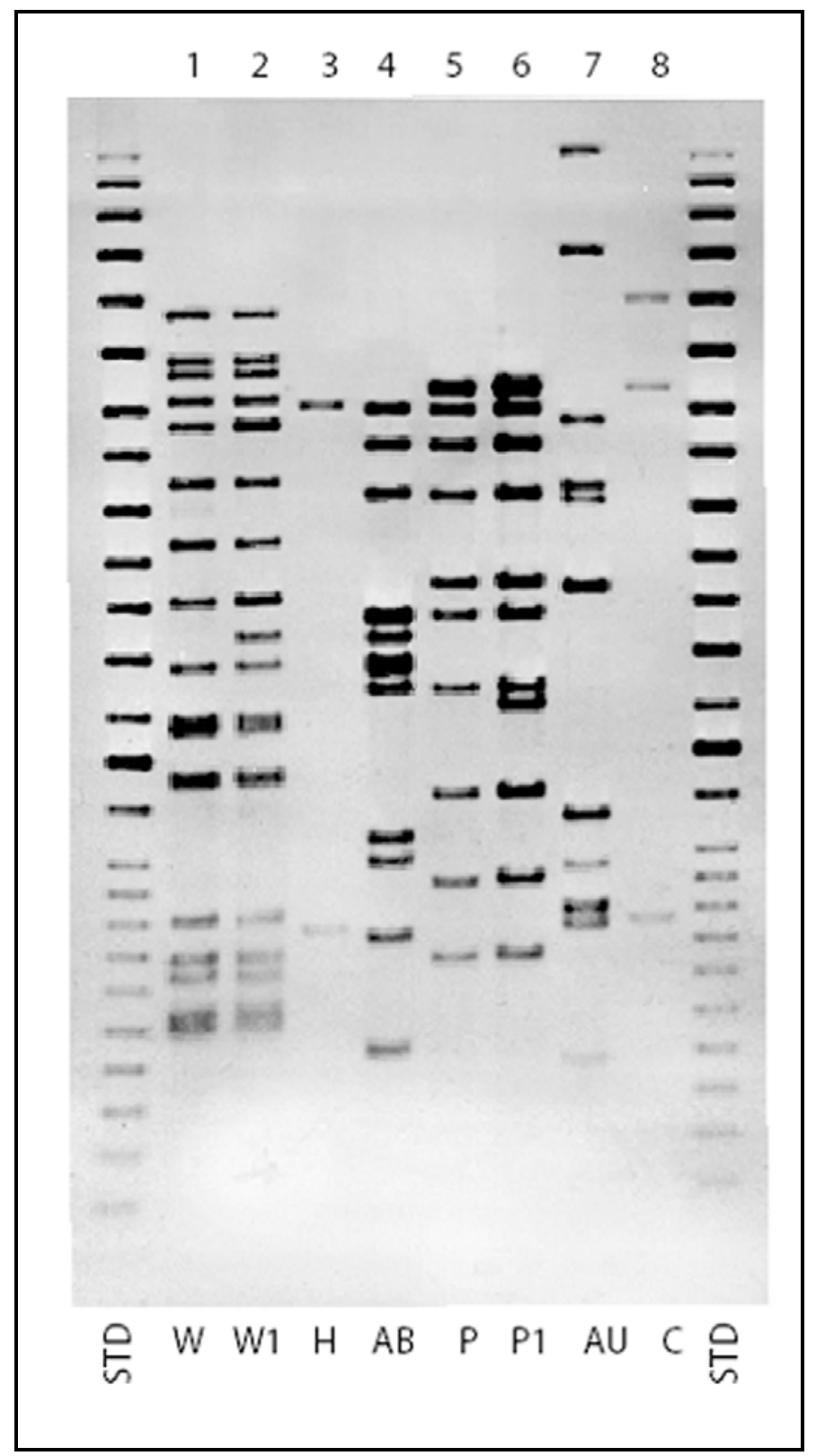

Figure 2. Insertion sequence (IS) 6110 Southern blot hybridization patterns for major multidrug-resistant Mycobacterium tuberculosis strains, New York City, 1995-1997. STD, standard. 
TUBERCULOSIS GENOTYPING NETWORK

Table 3. Risk factors associated with clustering of multidrug-resistant tuberculosis cases, New York City, 1995-1997 $(n=234)^{a}$

\begin{tabular}{|c|c|c|c|c|}
\hline \multirow[b]{2}{*}{ Characteristic } & Clustered $(\mathrm{n}=153)$ & Nonclustered $(\mathrm{n}=81)$ & Crude & \multirow[b]{2}{*}{$95 \% \mathrm{CI}$} \\
\hline & No. $(\%)$ & No. $(\%)$ & OR & \\
\hline Median age in yr (range) & $41(5-85)$ & $42(22-77)$ & 0.99 & $0.98,1.02$ \\
\hline U.S.-born & $120(79.0)$ & $42(51.9)$ & 3.48 & $1.94,6.25$ \\
\hline Median years of residence in United States ${ }^{\mathrm{b}}$ & $12(0-47)$ & $6.5(0-24)$ & 1.09 & $1.02,1.16$ \\
\hline Positive & $96(62.8)$ & $29(35.8)$ & 2.81 & $1.52,5.22$ \\
\hline Negative & $40(26.1)$ & $34(42.0)$ & 1.00 & \\
\hline Unknown & $17(11.1)$ & $18(22.2)$ & 0.80 & $0.36,1.80$ \\
\hline \multicolumn{5}{|l|}{ Race/ethnicity } \\
\hline White non-Hispanic & $26(17.0)$ & $10(12.4)$ & 1.27 & $0.53,3.06$ \\
\hline Health-care worker & $12(7.8)$ & $2(2.5)$ & 3.36 & $0.73,15.40$ \\
\hline Homeless & $22(14.4)$ & $5(6.2)$ & 2.55 & $0.92,7.02$ \\
\hline Injection drug use ${ }^{c}$ & $24(15.7)$ & $8(9.9)$ & 1.70 & $0.73,3.97$ \\
\hline Prior treatment history & $10(6.5)$ & $12(14.8)$ & 0.40 & $0.17,0.98$ \\
\hline \multicolumn{5}{|l|}{ Having epidemiologic link ${ }^{\mathrm{d}}$} \\
\hline Nosocomial & $7(4.6)$ & $0(0)$ & 2.97 & $1.02,9.26$ \\
\hline Community & $18(11.8)$ & $5(11.1)$ & & \\
\hline
\end{tabular}

demiologic links for nosocomial transmission were found for only two of the seven. Patients with this strain were identified from four of the city's five boroughs. The epidemiology of these clusters has been described in greater detail after the institutional outbreaks (22).

The only difference between the P and P1 strains is that the P1 strain has an additional band. Both strains have been nosocomially transmitted in one institution in New York City (7). Nine of the 13 patients with the P strain and all 4 with the P1 strain were living in the same borough as the institution where this outbreak was identified. However, epidemiologic links were identified for only one patient. Patients in these clusters were much less likely to be HIV infected than the other clustered patients $(29 \%$ vs. $67 \%, \mathrm{p}=0.002)$.

The $\mathrm{H}$ strain, the other major cluster, was also associated with a nosocomial outbreak in an institution in New York City
(8). During the study period, patients with this strain were identified from all the city's boroughs. Two patients with this strain had epidemiologic links.

\section{Discussion}

During the 3-year period, 241 (3.9\%) of all 6,228 TB cases in New York City and 241 (4.9\%) of all 4,995 M. tuberculosis culture-positive patients with susceptibility had MDR strains. MDRTB patients were more likely to have acid-fast bacilli visible on microscopic examination of respiratory specimens and thus were more infectious. MDRTB was more common in patients who were born in the United States, HIV infected, non-Asian, or health-care workers. The finding of greater prevalence of HIV infection in MDRTB patients compared with non-MDRTB patients is likely due to several reasons. The initial outbreaks during which these strains were transmit- 
ted mostly involved HIV-infected persons (1-8). A large number of HIV-infected patients were likely infected in those outbreaks. HIV-infected patients progress from infection to disease at a much higher rate than non-HIV-infected persons.

Most patients in this study had primary MDRTB caused by a few strains. The proportion of patients clustered in this cohort is much higher than in previously reported New York City patients. Few demographic and clinical characteristics were associated with clustering. According to previous citywide surveys of all patients who had cultures that were positive for TB, the proportions of clustered patients who had TB were $37 \%$ in 1991 and $32.4 \%$ in $1994(10,11)$. Another investigation from one hospital in the city found similar results for patients during 1989-1991 (9). A more recent survey from 1997 in New York City found that, for persons born in the United States, the proportion clustered had not decreased (23). In all these surveys, MDRTB was associated with clustering in multivariate analyses. The higher proportion of MDRTB clustering seen in this study cannot be explained by the exclusion of low-band patients in previous citywide surveys. When we exclude low-band patients from our analysis, we still have a similar proportion of clustering (128 [64.6\%] of 198 isolates with less than five bands). Our proportion of MDRTB clustering is also higher than that reported from other U.S. cities and other industrialized countries, where approximately $18 \%$ to $49 \%$ of clustering has been observed (24-28). However, few patients in these studies had MDRTB. The inclusion of MDRTB patients only in this study may have contributed to this difference. A study conducted during 1995 to 1997 by Moro et al. in Italy showed $74.2 \%$ clustering among MDRTB patients, compared with 39.3\% among non-MDR cases (29). Our study reiterates that a few, highly resistant strains were transmitted widely in New York City during the late 1980s and early 1990s.

Strains $\mathrm{W}, \mathrm{W} 1, \mathrm{P}, \mathrm{P} 1$, and $\mathrm{H}$ were transmitted in the early 1990s during the period of MDRTB outbreaks in New York City because five of the eight major clustered strains were associated with hospital outbreaks during that time (1-8). Few patients in this cohort had epidemiologic links, but most of these links were to patients whose diseases were diagnosed before the study period. Most health-care workers (10 of 14 with DNA results) had one of the known outbreak strains, but only 2 could be linked to facilities where nosocomial transmission occurred.

In addition to the nosocomially transmitted strains, we identified a large cluster that may have been transmitted in a community of persons who were HIV infected, homeless, and drug users. Before this study period, at least 14 additional MDRTB patients with this strain had been identified and confirmed by genotyping from 1989 through 1994. Six of these patients were from the same borough, and four were from the same health district as many of the patients in 1995-1997. This strain was transmitted over many years among drug users who were frequenting crack dens in the same neighborhood.
Since many of these venues were closed in the late 1990s, this social group was disrupted, and transmission was interrupted. The $\mathrm{AB}$ strain has been found in only two new patients during 1998-2001, one in 1998 and the other in 2001. The patient from 2001 had epidemiologic links to a patient from 1995.

Five patients had the $\mathrm{C}$ strain, which has three IS6110 copies. This M. tuberculosis strain is the most common in the city. Most of the $\mathrm{C}$ strains in the city share the same spoligotype and pTBN12-based RFLP pattern and are clonal (30, New York City Department of Health and Public Health Research Institute, unpub. data). Most of the $\mathrm{C}$ strains have been drugsusceptible; however, we identified $\mathrm{C}$ strains with varying drug-resistant patterns, occasionally in clusters (30, New York City Department of Health and Public Health Research Institute, unpub. data). The MDR strains in this period appear to be a recent cluster, or each may have acquired drug resistance separately.

MDRTB continues to decline in New York City at a rapid rate, with only 38,31 , and 25 new cases identified in 1998, 1999 , and 2000, respectively (31). However, most of the major strains found in this investigation continued to be identified in new MDRTB patients in New York City from 1998 through 2001 (New York City Department of Health and Public Health Research Institute, unpub. data). Most nonclustered patients had primary drug-resistant TB. The improved Tuberculosis Control Program, which was implemented in 1992 with aggressive case management and direct observation of anti-TB therapy for most patients, quickly curtailed the development of newly acquired drug resistance. Since primary and acquired drug resistance and MDRTB, in particular, were prevalent before 1995 (32,33), many MDRTB strains likely were disseminated in the community because most patients in this cohort with unique strains had no histories of prior treatment.

In this study, we may have underestimated the number of cases that had nosocomial and community epidemiologic links. We did not use medical record reviews of hospitalizations before the diagnosis of MDRTB for all the patients to identify potential nosocomial exposures. Many patients died before identification of MDRTB; therefore, interviews could not be conducted to identify potential nosocomial and community exposures before diagnosis of TB. The outbreaks associated with the $\mathrm{W}$ and $\mathrm{W} 1$ strains were well investigated and publicized, and staff were aware of the locations of the outbreak hospitals. This fact may have allowed for easier identification of epidemiologic links in these patients. In the $\mathrm{AB}$ community outbreak cluster, most epidemiologic links were identified from the detailed interviews with the few patients who were still alive. Traditional contact investigations did not identify these links in this subpopulation. This observation underscores that other methods, such as ongoing surveillance for unusual patterns of disease and unusual patient characteristics, should also be used to identify possible transmission in the community. Prospective DNA typing of all isolates can also supplement traditional contact investigation methods. 
The molecular analysis of the MDRTB strains in New York City during these years demonstrated that the improved Tuberculosis Control Program has reduced dramatically the transmission of these strains. These investigations have also established important baseline data for the study of the epidemiology of MDRTB over the next decades.

\section{Acknowledgments}

We acknowledge the work of the following Multi-Drug Resistant Tuberculosis Coordinators in the Tuberculosis Control Program for the case management and epidemiologic assessment of patients: Tripti Bhattacharjee, Sharif Choudhury, Anatole Hounnou, Cliff Johnson, Angel La Paz, Dileep Sarecha, and Iris Winter. In addition, we thank Cindy Driver, Paula Fujiwara, and Thomas Frieden for their thoughtful review of the manuscript.

The Public Health Research Institute TB Center and the Wadsworth Center received funds for this project from the Centers for Disease Control and Prevention, National Tuberculosis Genotyping and Surveillance Network.

Dr. Munsiff has been the director of the New York City Tuberculosis (TB) Control Program since December 2000, and she has been a medical officer in the Division of TB Elimination, National Center for HIV, STD, and TB Prevention, Centers for Disease Control and Prevention since November 2001. Her research interests include the epidemiology and clinical aspects of TB, particularly as manifested in HIV-infected persons, epidemiology and treatment of drug-resistant $\mathrm{TB}$, and program evaluation.

\section{References}

1. Centers for Disease Control and Prevention. Nosocomial transmission of multidrug-resistant tuberculosis among HIV-infected persons-Florida and New York, 1988-1991. MMWR Morb Mortal Wkly Rep 1991;40:589-91.

2. Valway SE, Richards SB, Kovacovich J, Greifinger RB, Crawford JT, Dooley SW. Outbreak of multidrug-resistant tuberculosis in a New York State Prison. Am J Epidemiol 1994;140:113-22.

3. Coronado VG, Beck-Sague CM, Hutton MD, Davis BJ, Nicholas P, Villareal C, et al. Transmission of multidrug-resistant Mycobacterium tuberculosis among persons with human immunodeficiency virus infection in an urban hospital: epidemiological and restriction fragment length polymorphism analysis. J Infect Dis 1993;168:1052-5.

4. Frieden TR, Sherman LF, Maw KL, Fujiwara PI, Crawford JT, Nivin B, et al. A multi-institutional outbreak of highly drug resistant tuberculosis. JAMA 1996;276:1229-35.

5. Nivin B, Nicholas P, Gayer M, Frieden TR, Fujiwara P. A continuing outbreak of multidrug-resistant tuberculosis, with transmission in a hospital nursery. Clin Infect Dis 1998;26:303-7.

6. Small PM, Shafer RW, Hopewell PC, Singh SP, Murphy MJ, Desmond E, et al. Exogenous reinfection with multidrug-resistant Mycobaterium tuberculosis in patients with advanced HIV infection. N Engl J Med 1993;328:1137-44.

7. Shafer RW, Small PM, Larkin C, Singh SP, Kelly P, Sierra MF, et al. Temporal trends and transmission patterns during the emergence of multidrug-resistant tuberculosis in New York City: a molecular epidemiological assessment. J Infect Dis 1995;171:170-6.

8. Pearson ML, Jereb JA, Frieden TR, Crawford JT, Davis BJ, Dooley SW, et al. Nosocomial transmission of multidrug-resistant Mycobacterium tuberculosis: a risk to patients and health-care workers. Ann Intern Med 1992;117:191-6.
9. Alland D, Kalkut GE, Moss AR, McAdam RA, Hahn JA, Bloom BR, et al. Transmission of tuberculosis in New York City—an analysis by DNA fingerprinting and conventional epidemiologic methods. N Engl J Med 1994;330:1710-6.

10. Frieden TR, Woodley CL, Crawford JT, Lew D, Dooley SM. The molecular epidemiology of tuberculosis in New York City: the importance of nosocomial transmission and laboratory error. Tuber Lung Dis 1996;77:407-13.

11. Sachdev PS, Fujiwara PI, Cook S, Kreiswirth BN, Frieden TR. Epidemiology of tuberculosis in NYC, April 1994: Determinants of recent transmission. In: Abstracts of the American Thoracic Society: San Francisco, California; 1997 May 16-21; Abstract A224. New York, New York: American Thoracic Society, 1997.

12. New York City Tuberculosis Control Program. 1994 Annual Summary. New York: New York City Department of Health;1995.

13. Frieden TR, Fujiwara PI, Washko RM, Hamburg MA. Tuberculosis in New York City - turning the tide. N Engl J Med 1995;333:229-33.

14. Hawkins JE, Wallace RJ Jr, Brown BA. Antibacterial susceptibility tests: mycobacteria. In: Balows A, Hausler WJ Jr, Herrmann K, Isenberg HD, Shadomy HJ, editors. Manual of clinical microbiology. 5th ed. Washington: American Society for Microbiology; 1991. p. 1138-52.

15. Kent PT, Kubica GP. Public health mycobacteriology: A guide for the level III laboratory. Atlanta: Department of Health and Human Services; 1985.

16. van Embden JD, Cave MD, Crawford JT, Dale JW, Eisenbach KD, Gicquel P, et al. Strain identification of Mycobacterium tuberculosis by DNA fingerprinting: recommendations for a standardized methodology. J Clin Microbiol 1993;31:406-9.

17. Kreiswirth BN, Moss A. Genotyping multidrug-resistant M. tuberculosis in New York City. In: Rom WN, Garay SM, editors. Tuberculosis. Boston: Little, Brown, and Company, Inc; 1996. p.199-209.

18. Groenen PM, Bunschchoten AE, van Soolingen D, van Embden JD. Nature of DNA polymorphism in the direct repeat cluster of Mycobacterium tuberculosis; application for strain identification by a novel typing method. Mol Microbiol 1993;10:1057-65.

19. Goyal M, Saunders MA, van Embden JDA, Young DB, Shaw RJ. Differentiation of Mycobacterium tuberculosis isolates by spoligotyping and IS6110 restriction fragment length polymorphism. J Clin Microbiol 1997;35:647-51.

20. Ramaswamy S, Musser JM. Molecular genetic basis of antimicrobial agent resistance in Mycobacterium tuberculosis: 1998 Update. Tuber Lung Dis 1998;79:3-29.

21. Dale JW, Brittan D, Cataldi AA, Cousins D, Crawford JT, Driscoll J, et el. Spacer oligonucleotide typing of bacteria of the Mycobacterium tuberculosis complex: recommendation for standardized nomenclature. Int $\mathrm{J}$ Tuberc Lung Dis 2001;5:216-19.

22. Munsiff SS, Nivin B, Sacajiu G, Bassoff T, Mathema B, Bifani P, et el. Evolution of a highly drug resistant strain of tuberculosis in New York City. In: Abstracts of the American Society of Microbiology Tuberculosis: past, present and future; New York, New York, 2000 June 20-24; Abstract 90. Washington: American Society of Microbiology; 2000.

23. Sachdev PS, Bassoff T, Kreiswirth B, Cook S, Munsiff SS, Fujiwara PI. Molecular epidemiology of tuberculosis in NYC: an ongoing survey, April 1997. Resistant strain of tuberculosis in New York City. In: Abstracts of the American Thoracic Society: San Diego, California; 1999 April 23-28; Abstract A904. New York: American Thoracic Society; 1999.

24. Bauer J, Yang Z, Poulsen S, Andersen AB. Results from 5 years of nationwide DNA fingerprinting of Mycobacterium tuberculosis complex isolates in a country with a low incidence of $M$. tuberculosis infection. J Clin Microbiol 1998;36:305-8.

25. Dahle UR, Sandven P, Heldal E, Caugant DA. Molecular epidemiology of Mycobacterium tuberculosis in Norway. J Clin Microbiol 2001;39:1802-7.

26. Gutierrez MC, Vincent V, Aubert D, Bizet J, Gaillot O, Lebrun L, et al. Molecular fingerprinting of Mycobacterium tuberculosis and risk factors for tuberculosis transmission in Paris, France, and surrounding area. J Clin Microbiol 1998;36:486-92. 
27. Jasmer RM, Hahn JA, Small PM, Daley CL, Behr MA, Moss AR, et al. A molecular epidemiologic analysis of tuberculosis trends in San Francisco, 1991-1997. Ann Intern Med 1999;130:971-8.

28. van Soolingen D, Borgdorff MW, de Haas PEW, Sebek MMGG, Veen J, Dessens M, et al. Molecular epidemiology of tuberculosis in the Netherlands: a nationwide study from 1993-1997. J Infect Dis 1999;180:726-36.

29. Moro ML, Salamina G, Gori A, Penati V, Sacchetti R, Mezzetti F, et al. Two-year population-based molecular epidemiological study of tuberculosis transmission in the Metropolitan area of Milan, Italy. Eur J Clin Microbiol Infect Dis 2002;21:114-22.

30. Friedman CR, Quinn GC, Kreiswirth BN, Perlman DC, Berger J, Riley $\mathrm{LW}$, et al. Widespread dissemination of a drug-susceptible strain of $\mathrm{Myco}-$ bacterium tuberculosis. J Infect Dis 1997;176:478-84.
31. New York City Tuberculosis Control Program. 2000 annual summary. New York: New York City Department of Health; 2001.

32. Frieden TR, Sterling T, Pablos-Mendez A, Kilburn JO, Cauthen GM, Dooley SW, et al. The emergence of drug-resistant tuberculosis in New York City. N Engl J Med 1993;328:521-6.

33. Fujiwara PI, Cook SV, Rutherford CM, Crawford JT, Glickman SE, Kreiswirth BN, et al. A continuing survey of drug-resistant tuberculosis, New York City, April 1994. Arch Intern Med 1997;157:531-6.

Address for correspondence: Sonal S. Munsiff, New York City Department of Health and Mental Hygiene, 125 Worth St., Room 216, CN74, New York, NY 10013, USA; fax: 212-788-9836; e-mail: smunsiff@health.nyc.gov

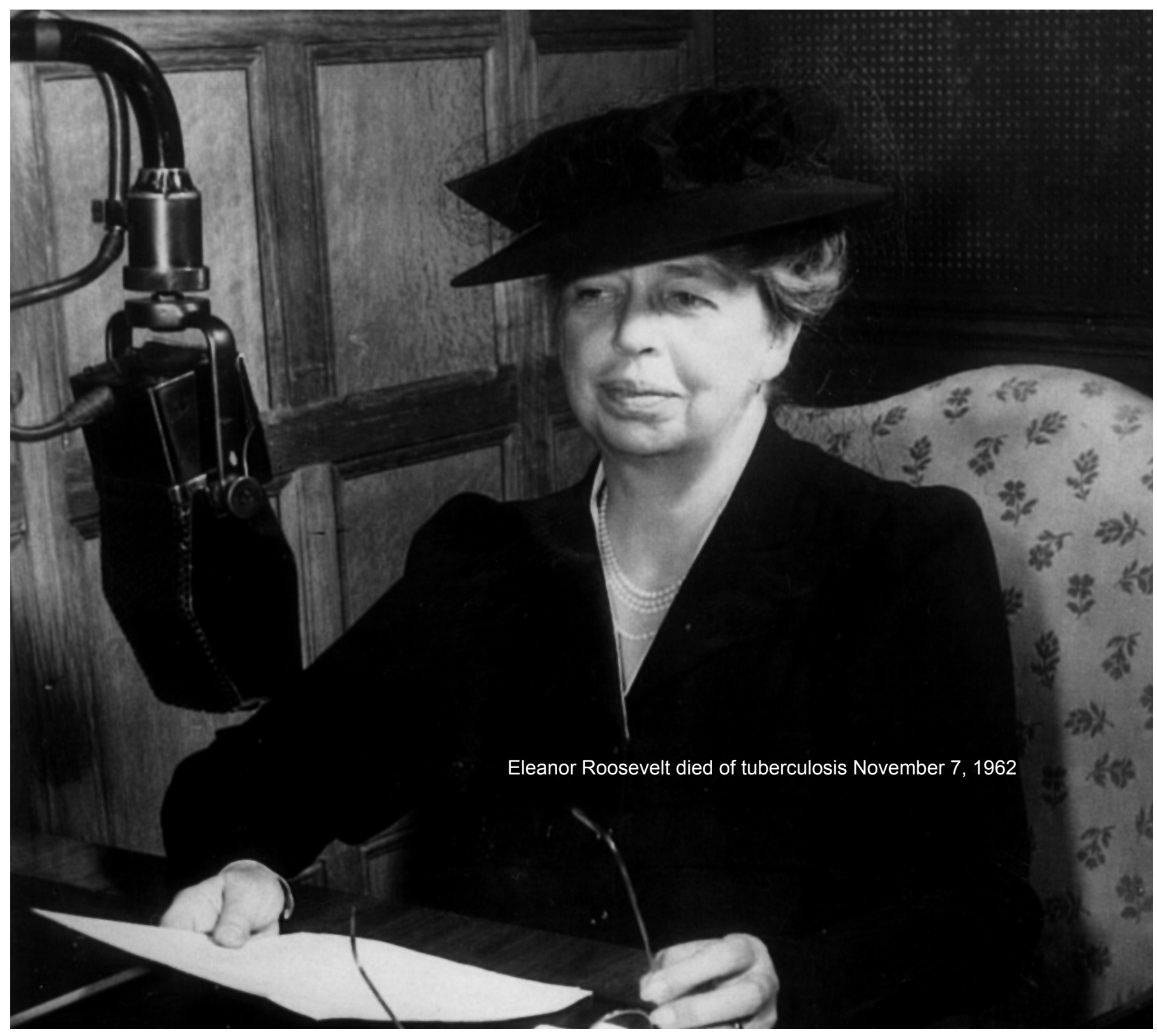

